\title{
Resistencia compresiva vidrio ionómero lonofil Molar® y Vitremer® según tiempo de exposición en saliva artificial
}

\author{
Compressive strength of glass ionomer lonofil Molar ${ }^{\circledR}$ and \\ Vitremer ${ }^{\circledR}$ according to exposure time in artificial saliva
}

\author{
Hernández González $\mathrm{R}^{1}$, Moraga Castillo $\mathrm{R}^{2}$, Velásquez Castilla $\mathrm{M}^{3}$, Gutiérrez Flores $\mathrm{F}^{4}$
}

\begin{abstract}
RESUMEN
Objetivo: El conocimiento sobre las propiedades mecánicas de los materiales dentales es fundamental para una correcta indicación y funcionamiento en la cavidad oral, permitiéndole al profesional optar por el que presente mejor comportamiento durante la masticación. El objetivo de este estudio fue evaluar la resistencia a la compresión de lonofil Molar ${ }^{\circledR}$ y Vitremer®, según tiempo de exposición en saliva artificial. Materiales y Métodos: Se prepararon 5 muestras para cada material evaluadas a los tiempos 0,168 y 504 horas en saliva artificial a $37^{\circ} \mathrm{C}$, según las especificaciones propuestas por la norma ANSI/ADA $n^{\circ} 66$. La resistencia a la compresión se determinó sometiendo las muestras a cargas en un equipo de ensayo de fuerzas Instron $®$ a una velocidad de carga de $1 \mathrm{~mm} / \mathrm{min}$. Se realizó una prueba de homogeneidad de varianzas, la normalidad se determinó mediante Kolmogorov-Smirnov, y posteriormente un ANOVA. Se realizó el test de Tukey para determinar si existió diferencia significativa entre variables. Resultados: La resistencia compresiva de Vitremer no presentó diferencias estadísticamente significativas en el tiempo ( $p=0.282$ ), a diferencia de lonofil Molar, que sí presentó diferencias entre los distintos tiempos ( $p=0.011)$. Además en las muestras sin sumergir, no hubo diferencia estadísticamente significativa entre materiales $(p=0.091)$, en cambio sí existió diferencia al cabo de una y tres semanas de exposición ( $p=0$ ). Conclusión: El vidrio ionómero Vitremer presenta mayor resistencia compresiva a lo largo del tiempo, sin presentar alteraciones significativas en el tiempo al ser inmerso en saliva, a diferencia del lonofil Molar que disminuyó significativamente su resistencia en las mismas condiciones.
\end{abstract}

Rev. Clin. Periodoncia Implantol. Rehabil. Oral Vol. 6(2); 75-77, 2013.

Palabras clave: Cemento de vidrio ionómero, resistencia compresiva, saliva artificial.

\section{ABSTRACT}

Objective: Understanding the mechanical properties of dental materials is essential for proper indication and a correct functioning in the oral cavity, as it allows the dentist to choose the material that presents better performance during mastication. The aim of this study was to evaluate the compressive strength of lonofil Molar and Vitremer, according to the exposure time in artificial saliva. Materials: 5 samples were prepared for each material evaluated at 0, 168 and 504 hours in artificial saliva at $37^{\circ}$ Celsius, according to the specifications suggested by ANSI/ADA specification $\mathrm{N}^{\circ} 66$. The compressive strength was determined by subjecting the samples to an Instron strength-testing machine at a load speed of $1 \mathrm{~mm} / \mathrm{min}$. A test of homogeneity of variance was conducted; normality was determined by Kolmogorov-Smirnov, and ANOVA. Tukey's test was performed to determine if significant differences existed between variables. Results: Vitremer compressive strength did not show statistically significant differences over time $(p=0.282)$, unlike lonofil Molar, which did present differences between times $(p=0.011)$. Besides, there were no statistically significant differences in the samples without submerging $(p=0.091)$, unlike after one to three weeks of exposure, were a difference did exist $(p=0)$. Conclusion: Vitremer has greater compressive strength over time, without showing significant changes in time after being immersed in saliva, unlike lonofil Molar, whose resistance decreased significantly under the same conditions.

Rev. Clin. Periodoncia Implantol. Rehabil. Oral Vol. 6(2); 75-77, 2013.

Key words: Glass ionomer cement, compressive strength, artificial saliva.

\section{INTRODUCCIÓN}

Desde hace varias décadas que las principales empresas dedicadas a la elaboración de materiales dentales investigan nuevas tecnologías, con el fin de mejorar las propiedades de éstos. Es así como se han enfocado principalmente en el estudio y desarrollo de dos áreas: la estética y la biomecánica. Para evaluar esta última, son comunes las pruebas mecánicas, que permiten estudiar cómo reaccionan los materiales frente a distintas fuerzas ${ }^{(1,2)}$.

Los cementos de vidrio ionómero fueron desarrollados e introducidos en los años $70^{(3)}$, componiéndose principalmente de vidrio, poliácidos y agua. El vidrio se presenta en forma de polvo y es capaz de liberar gran cantidad de iones calcio, aluminio y fluoruro ${ }^{(4)}$. Los poliácidos se presentan en forma de líquido, generalmente éste esta formado por ácido poliacrílico en solución acuosa, pero también puede utilizarse ácido tartárico, maléico o fosfórico. El último componente es el agua, que tiene la función de proporcionar el medio en que se realizan los intercambios iónicos ${ }^{(4)}$. Estos componentes se mezclan formando un cemento mediante una reacción ácido-base inmediata ${ }^{(4)}$. Actualmente también se ha incorporado resina, generando vidrios ionómeros de fotocurado, con el fin de suplir las desventajas del vidrio ionómero convencional(5), mejorando así sus propiedades mecánicas, estéticas( ${ }^{(3)}$ e hidrófobas debido a que los vidrios ionómeros convencionales experimentan solubilidad y desintegración en el medio bucal(6). Estas propiedades no son fáciles de determinar debido a factores como la composición de cada uno de los materiales de las distintas marcas comerciales, de su proceso de fabricación, del tamaño de las partículas de polvo, el tipo de vidrio ionómero, a la concentración y peso molecular del líquido y a la relación vidrio ionómero/resina ${ }^{(7)}$

El vidrio ionómero es un material muy utilizado en la

1. Cirujano Dentista. Facultad de Odontología, Universidad del Desarrollo. Concepción, Chile.

2. Ingeniero Civil Eléctrico. Facultad de Odontología, Universidad del Desarrollo. Concepción, Chile.

3. Cirujano Dentista. Diplomado (c) en Metodología de la Investigación Clínica. Facultad de Odontología, Universidad del Desarrollo. Concepción, Chile.

4. Ingeniero Civil Industrial. Magister (c) en Docencia Universitaria. Universidad del Desarrollo. Concepción, Chile. 
odontología actual debido a sus propiedades que lo hacen útil tanto en procedimientos de cementación como obturación. Sus principales indicaciones son como liner o base para restauraciones metálicas o de resinas compuestas; como material para reconstitución de muñones; como material de restauración y también para cementación de restauraciones rígidas ${ }^{(8)}$. Dentro de su uso como material de obturación, el VI se considera el material de elección para realizar la técnica de restauración atraumática (TRA) ${ }^{(9)}$. En ésta, se utiliza vidrio ionómero convencional para obturar cavidades realizadas manualmente ${ }^{(10)}$. De igual manera, se han reportado casos donde se ha utilizado un vidrio ionómero modificado con resina (VIMR) obteniendo resultados similares en cuanto a permanencia en el tiempo(11).

Durante la masticación actúan distintas fuerzas, como esfuerzos de tracción, flexión y compresión. El conocimiento sobre estos esfuerzos, en especial los de compresión, permitirá al profesional tomar la mejor decisión al momento de obturar una cavidad clase 1 ó 2 con vidrio ionómero en relación a su comportamiento biomecánico, evitando así el fracaso de dichas obturaciones a cargo de fuerzas masticatorias ${ }^{(4)}$.

A pesar de la existencia de éstos antecedentes, no hay evidencia de estudios que comparen el comportamiento biomecánico de un vidrio ionómero convencional y otro modificado con resina tras la exposición a una solución de saliva artificial. Tanto lonofil Molar como Vitremer se pueden utilizar para la obturación de lesiones cariosas mediante la TRA, por lo que la comparación de sus propiedades mecánicas entregaría información para justificar el uso de un VIMR en ésta técnica. El presente trabajo tuvo como objetivo estudiar y comparar el comportamiento de la resistencia compresiva de dos tipos de vidrios ionómeros, uno convencional y uno modificado con resina, después de ser sometidos a una solución de saliva artificial a $37^{\circ} \mathrm{C}$, simulando las condiciones intraorales de una restauración preparada con dicho material.

\section{MATERIALES Y MÉTODOS}

Se realizó un estudio de prueba de materiales. Los materiales estudiados fueron lonofil Molar ${ }^{\circledR}$ (VOCO GmbH, Cuxhaven, Germany) y Vitremer ${ }^{\circledR}(3 \mathrm{M}$ ESPE, St. Paul, MN, U.S.). La muestra fue determinada por las especificaciones propuestas por la norma ANSI/ADA número 66 para cementos de vidrio ionómero(12), con un $95 \%$ de confiabilidad, un error alfa de $5 \%$ y una heterogeneidad de $50 \%$. Dicha muestra consideró 5 especímenes de lonofil Molar y 5 de Vitremer para cada tiempo de exposición ( $\mathrm{t} 0=0 \mathrm{~h}, \mathrm{t} 1=168 \mathrm{~h}$ y t2= $504 \mathrm{~h}$ ), con un total de 30 muestras. Estas fueron fabricadas y preparadas según las especificaciones establecidas por la norma y las instrucciones de cada fabricante.

Las muestras se fabricaron en un molde preformado de $6 \mathrm{~mm}$ de alto por $3 \mathrm{~mm}$ de diámetro de acuerdo a los estándares establecidos, éstas fueron inmersas en tubos de ensayos con $5 \mathrm{ml}$ de solución de saliva artificial, tapados con algodón hidrófobo y almacenadas en una estufa de incubación a $37^{\circ} \mathrm{C}^{(2)}$. La solución de saliva artificial se preparó con $9.8 \mathrm{~g}$ bicarbonato, $7 \mathrm{~g}$ fosfato ácido de sodio heptahidratado, $0.57 \mathrm{~g}$ cloruro de potasio, $0.47 \mathrm{~g}$ cloruro de sodio, $0.04 \mathrm{~g}$ cloruro de calcio y 0.12 $\mathrm{g}$ sulfato de magnesio por litro de solución.

Las pruebas mecánicas se realizaron mediante el uso de una máquina de ensayos universales Instron (Instron ${ }^{\circledR}$, Barcelona, España). 5 muestras de cada material fueron testeadas sin ser inmersas en saliva. Las 20 restantes fueron inmersas en una solución de saliva artificial, 10 de estas fueron testeadas a las 168 horas después de inmersas y las 10 restantes a las 504 horas después de ser sumergidas. La recolección de datos se realizó a través del software Bluehill Lite asociado a la máquina de ensayos universales, luego los datos fueron tabulados en el software Microsoft Excel y posteriormente se realizó el ingreso de la base de datos al software estadístico SPSS. Se compararon los resultados entre las muestras del mismo material y en los distintos tiempos de medición, para esto se realizó una prueba de homogeneidad de varianzas, un análisis de normalidad (Kolmogorov-Smirnov) y un ANOVA. Posteriormente, se realizó el test de Tukey para determinar si existió diferencia significativa entre variables. Además, se incorporaron medidas de tendencia central como media, mediana, desviación estándar, mínimo y máximo.

\section{RESULTADOS}

Se evaluó la resistencia compresiva de 15 muestras de Vitremer y 15 muestras de lonofil Molar, estudiando el efecto de la inmersión en saliva sobre dicha resistencia. Los resultados obtenidos por Vitremer se pueden observar en la Tabla 1. Ionofil Molar presentó un comportamiento inferior (Tabla 2) comparado con Vitremer en todos los tiempos estudiados (Figura 1).

De acuerdo a lo resultados obtenidos, se determinó que Vitremer no presentó diferencias estadísticamente significativas en su resistencia a la compresión entre los distintos tiempos $(p=0.282)$, mientras lonofil Molar presentó diferencias significativas $(p=0.011)$.

En las muestras sin sumergir en saliva, no hubo una diferencia estadísticamente significativa entre ambos materiales $(p=0.091)$, pero sí existió diferencia entre estos al cabo de una y tres semanas de exposición $(p=0.000)$.

Tabla 1. Medidas de tendencia central y dispersión de la resistencia compresiva (MPa) de Vitremer® en los distintos tiempos de estudio.

\begin{tabular}{|cccccc|}
\hline Tiempo & Media & Mediana & $\begin{array}{c}\text { Desviación } \\
\text { estándar }\end{array}$ & Mínimo & Máximo \\
\hline t0 & 137.85 & 129.86 & 64.46 & 48.71 & 222.94 \\
t1 & 127.66 & 124.4 & 16.59 & 110.09 & 153.27 \\
t2 & 97.06 & 98.97 & 19.01 & 73.63 & 122 \\
\hline
\end{tabular}

Tabla 2. Medidas de tendencia central y dispersión de la resistencia compresiva (MPa) de lonofil Molar® en los distintos tiempos de estudio.

\begin{tabular}{|cccccc|}
\hline Tiempo & Media & Mediana & $\begin{array}{c}\text { Desviación } \\
\text { estándar }\end{array}$ & Mínimo & Máximo \\
\hline t0 & 76.60 & 85.41 & 30.53 & 39.76 & 116.64 \\
t1 & 45.26 & 45.15 & 12.44 & 25.19 & 57.39 \\
t2 & 30.05 & 30.13 & 12.76 & 17.13 & 44.15 \\
\hline
\end{tabular}

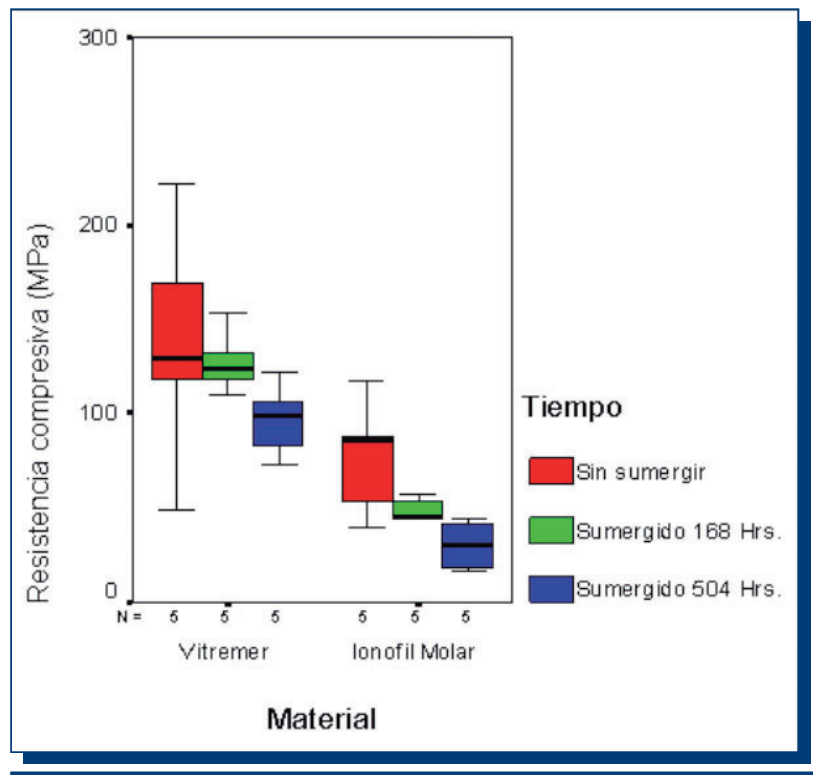

Figura 1. Diagrama de cajas de la resistencia compresiva (MPa) de Vitremer y lonofil Molar en los distintos tiempos de estudio.

\section{DISCUSIÓN}

Los resultados obtenidos permiten determinar que Vitremer presenta mayor resistencia a la compresión frente a la exposición en saliva artificial, sin presentar diferencias significativas a lo largo del tiempo, por lo tanto se comportará mejor en relación a restauraciones clase 1 y 2 donde el principal esfuerzo masticatorio es la compresión ${ }^{(13)}$. Estudios concuerdan en que la incorporación de materiales más resistentes a la 
fórmula base, como resina o metales, favorece la respuesta del vidrio ionómero frente a los esfuerzos compresivos ${ }^{(14)}$. Por otro lado, lonofil Molar presentó una significativa disminución de su resistencia después de ser inmerso en saliva, quedando en evidencia su hidrosolubilidad y la alteración que sufren sus propiedades mecánicas al ser expuesto a un medio acuoso.

Estos antecedentes son comparables con estudios realizados con anterioridad, donde se comprobaron las propiedades biomecánicas de distintas marcas de vidrio ionómero, entre ellas Vitremer, tras ser inmersos en agua destilada ${ }^{(15)}$. En este caso, Xie concluye que Vitremer presenta valores más altos de resistencia, aunque en el estudio también se comprueban otros parámetros biomecánicos como la resistencia flexural o a la tensión, por lo que en futuras investigaciones también se debería estudiar como se modifican éstas propiedades frente a la exposición en saliva. En cuanto a lonofil Molar, no hay trabajos previos que estudien la resistencia compresiva de este ionómero vítreo ya sea expuesto en una solución de saliva artificial o no, por lo que futuros estudios deben considerar pruebas de este biomaterial.

A pesar de quedar en evidencia los cambios experimentados por los vidrio ionómero en el estudio frente a la exposición en saliva, el agua juega un rol importante en la reacción ácido-base que ocurre al mezclar el polvo de vidrio con el ácido poliacrílico incluido en el líquido ${ }^{(4)}$. En las primeras etapas del fraguado, el agua proveniente de la parte líquida se incorpora totalmente en la estructura del cemento, pero al avanzar el proceso de fraguado se debe proteger al vidrio ionómero de ésta, para evitar procesos de disolución ${ }^{(4)}$. Finalmente, cuando el cemento ya ha alcanzado su estado sólido, la pérdida de agua puede deshidratarlo otorgándole un aspecto tiza ${ }^{(16)}$.

Para evitar la pérdida o exceso de agua en los ionómeros vítreos se han incorporado protectores insolubles en la presentación comercial de algunos vidrio ionómero de fotocurado, como Vitremer, siendo considerados como el mejor agente protector de superficie. Estudios avalan esta propiedad, demostrando que estos agentes limitan la absorción de agua por parte de los cementos $^{(16)}$. Seria de gran valor clínico, que en estudios posteriores se realizara una prueba de resistencia a lonofil Molar aplicando una capa protectora insoluble sobre su superficie, para así determinar si es posible mantener las propiedades biomecánicas del material a lo largo del tiempo.

Es posible señalar que Vitremer posee mejor capacidad de tolerar la exposición en saliva en el tiempo, ya que no presentó diferencias significativas en su resistencia compresiva al ser expuesto en una solución de saliva artificial. Esto se contrasta con la respuesta que tuvo lonofil Molar, ya que se generaron diferencias en su comportamiento frente al esfuerzo de compresión.

\section{CONFLICTO DE INTERESES} ningún tipo.
Los autores declaran no presentar conflictos de interés de

\section{REFERENCIAS BIBLIOGRÁFICAS}

1. Yap AUJ, Pek YS, Cheang P. Physico-mechanical properties of a fast-set highly viscous GIC restorative. J Oral Rehabil, 2003; 30: 1-8.

2. Bresciani E, Barata T, Ticiane F, Adachi A, Terrin M, Navarro M. Compressive and diametral tensile strength of glass ionomer cements. Oral Sci, 2004; 12(4): 334-338.

3. Mallmann A, Oliveira A, Jane C, Amoedo R, Rocha VP, Jaques LB. Compressive strength of glass ionomer cements using different specimen dimensions. Braz Oral Res, 2007; 21(3): 204-208.

4. Lohbauer U. Dental glass ionomer cements as permanent filling materials? Properties, limitations and future trends. Materials, 2010; 3(1): 76-96.

5. De Guzman A. Evaluación clínica de un ionómero de vidrio modificado en odontopediatría. Acta Odontol Venez, 2001; 39(3): 54-68.

6. Mooney J. Ionómeros vítreos y compómeros. $3^{\text {era }}$ ed. En: Mooney J, Operatoria dental. Buenos Aires: Medico-Panamericana, 1999: 635-654.

7. Aratani M, Pereira AC, Correr-Sobrinho L, Sinhoreti MA, Consani S. Compressive strength of resin-modified glass ionomer restorative material: Effect of $\mathrm{P} / \mathrm{L}$ ratio and storage time. J Appl Oral Sci, 2005; 13(4): 356-359.

8. Lahoud Salem V. Cementos a base de vidrio ionómero. Odontol Sanmarquina, 1998; 1(1):47-49.

9. Tascón J. Restauración atraumática para el control de caries dental: Historia, características y aporte de la técnica. Rev Panam Salud Publica, 2005; 17(2): 110-115.
10. Frencken JE, Pilot T, Songpaisany $\mathrm{Y}$, Phantumvanit $\mathrm{P}$. Atraumatic restorative treatment (ART): Rationale, technique, and development. J Public Health Dent 1996; 56: 135-140.

11. Dülgergil CT, Soyman $M$, Civelek A. Atraumatic restorative treatment with resinmodified glass ionomer material: Short-term results of a pilot study. Med Princ Pract, 2005; 14(4): 277-280

12. ANSI/ADA specification no. 66 for dental glass ionomer cements. Council on dental materials, instruments, and equipment. J Am Dent Assoc, 1989; 119(1): 205.

13. Hegde MN, Hegde P, Bhandary S, Deepika K. An evaluation of compressive strength of newer nanocomposite: An in vitro study. J Conserv Dent, 2011; 14(1): 36-39.

14. Cho GC, Kaneko LM, Donovan TE, White SN. Diametral and compressive strength of dental core materials. J Prosthet Dent, 1999; 82(3): 272-276.

15. Xie D, Brantley WA, Culbertson BM, Wang G. Mechanical properties and microstructures of glass-ionomer cements. Dent Mater, 2000; 16(2): 129-138.

16. Nicholson JW, Wilson AD. The effect of storage in aqueous solutions on glassionomer and zinc polycarboxylate dental cements. J Mater Sci Mater Med, 2000; 11: $357-360$ 